\title{
Robin-type wall functions and their numerical implementation
}

\author{
S.V. Utyuzhnikov \\ School of Mechanical, Aerospace \& Civil Engineering, University of Manchester, Manchester, M60 1QD, UK
}

Available online 26 September 2007

\begin{abstract}
The paper is devoted to numerical implementation of the wall functions of Robin-type for modeling near-wall turbulent flows. The wall functions are based on the transfer of a boundary condition from a wall to some intermediate boundary near the wall. The boundary conditions on the intermediate boundary are of Robin-type and represented in a differential form. The wall functions are formulated in an analytical easy-to-implement form, can take into account the source terms, and do not include free parameters. The relation between the wall functions of Robin type and the theory of Calderon-Ryaben'kii's potentials is demonstrated. A universal robust approach to the implementation of the Robin-type wall functions in finite-volume codes is provided. The example of an impinging jet is considered.
\end{abstract}

() 2007 IMACS. Published by Elsevier B.V. All rights reserved.

Keywords: Wall functions; Robin boundary conditions; Potentials; Difference potential method; Nonlocal boundary conditions; Boundary condition transfer; Approximation; Turbulence; Impinging jet

\section{Introduction}

Turbulent near-wall flows appear in many industrial problems. It is well known that turbulence vanishes near a wall due to both the no-slip boundary condition for the velocity and the blocking effect caused by the wall. In the vicinity of the wall, there is a thin sublayer with predominantly molecular diffusion and viscous dissipation. The sublayer has a substantial influence upon the remaining part of the flow. An adequate resolution of the solution in the sublayer requires a very fine mesh because of the thinness of the sublayer and high gradients of the solution. As a result, up to $50 \%$ boundary-layer nodes are situated in the near-wall region [17]. It makes the model to be time consuming and often it is not suitable for real design.

The models that resolve the sublayer are called low-Reynolds-number (LR) models because of the low turbulent Reynolds number in the sublayer. In turn, the high-Reynolds-number (HR) models do not resolve the viscous sublayer. They significantly save computational efforts $[7,19]$. In the HR models, the boundary conditions or near-wall profiles are represented by wall functions. Wall functions are usually semi-empirical and have quite limited applications. A substantial disadvantage of many wall functions is a strong dependence on the near wall mesh used. To avoid this problem, the scalable wall functions are suggested in [11]. Wilcox assumes [30] that the pressure gradient must be taken into account to avoid the mesh dependence. Yet, the recently suggested adaptive wall functions [15] overcome this limitation by using look-up tables for turbulent quantities and skin friction. This approach is extended to separated flows in [17].

E-mail address: s.utyuzhnikov@manchester.ac.uk. 
In $[7,8]$ source terms, such as the pressure gradient, are taken into account in the wall functions via the approximate integration of the near-wall governing equations. The numerical comparisons $[7,8]$ showed that such advanced wall functions give substantially better prediction than the standard wall functions. In [8], the analytical wall functions are obtained by approximate integrating boundary-layer-type equations in the wall vicinity. The wall functions $[7,8]$ are only represented in a finite-difference form rather than a differential form. In computations, the numerical flux to the wall is taken into account by iterations as it is performed in the case of the standard wall functions.

The method of boundary condition transfer is suggested in [27,29]. The method allows us to transfer a boundary condition from the wall to some intermediate surface. The boundary condition is transfered either approximately (analytically) or exactly (numerically). The boundary conditions on the intermediate surface are always of Robin-type (or mixed type) and represented in a differential, mesh independent, form. These boundary conditions are interpreted as Robin-type wall functions (RWF). They are simultaneously set on both a function and its normal derivative. Therefore, the realization of RWF does not require any additional iterations between, for example, the velocity and skin friction. It is important to emphasize that this property of RWF brings an additional robustness to the algorithm of their implementation. Another advantage of RWF is related with their universal formulation for all dependent variables. It is also worth noting that the boundary conditions represented by RWF are similar to the "slip boundary condition" at the edge of the Knudsen-layer in aerodynamics.

RWF take into account the influence of the source terms in the governing equations in a unit manner. The location of the point, to which the boundary conditions are transferred, does not make any considerable effect on the mesh distribution nearby this point. The wall functions can be implemented in both finite-difference and finite-volume approximations. Tests for channel flow [28] and impinging jet [29] have shown promising results in the terms of both accuracy and efficiency gains. In comparison to the analytical and numerical wall functions $[7,8]$, the key advantages of RWF are related with their robust implementation and universal differential formulation.

In the paper below RWF are derived via the theory of Calderon-Ryaben'kii's potentials. From this theory it follows that RWF provide the sufficient and necessary condition for the functions to be extended to the domain of the main flow. They do not depend on either the external domain or the differential operator used there. Thus, RWF can be used not only for the Reynolds Averaged Navier-Stokes equations (RANS) but for Large Eddy Simulation (LES) as well. They can be realized in a separate routine which can be used in different RANS and LES codes. A universal approach to realizing RWF in existing finite-volume codes is provided. The approach is applicable to all governing equations in a unit manner. As an example, RWF are implemented in the $k-\epsilon$ model and applied for the axisymmetric impinging jet problem. A wide range of geometries and operating regimes for the impinging jet is considered. The computational results are compared against available experimental data. These test results supplement the results obtained in [29] for the impinging-jet test case to different geometries and operating regimes. The important conclusion from the calculations done is that the wall functions are applicable to completely different regimes and mesh sizes without the use of any free parameters.

\section{Robin-type wall functions}

To formulate RWF, first let us assume that a governing equation is written in the following form:

$$
L u \equiv\left(\mu u_{y}\right)_{y}=R_{h}(y), \quad 0 \leqslant y \leqslant y_{e},
$$

with Dirichlet boundary condition on the left-hand side:

$$
u(0)=u_{0} .
$$

It is to be noted here that the Dirichlet boundary condition is not a limitation for the use of RWF [27], and the case of general boundary conditions is considered in the next section. Eq. (1) represents the general form of the boundarylayer-type equation. The right-hand side $R_{h}$ is an appropriate source term including, e.g., the pressure gradient and convective terms in the momentum equation.

RWF are formulated as follows [27,29]:

$$
u\left(y^{*}\right)=u_{0}+f_{1} \frac{d u}{d y}\left(y^{*}\right)-\left(\int_{0}^{y^{*}} R_{h} d y\right) \frac{f_{2}}{y^{*} \mu\left(y^{*}\right)},
$$


where

$$
f_{1}=\int_{0}^{y^{*}} \frac{\mu\left(y^{*}\right)}{\mu(y)} d y, \quad f_{2}=y^{*} \int_{0}^{y^{*}} \frac{\mu\left(y^{*}\right)}{\mu(y)}\left(1-\frac{\int_{0}^{y} R_{h} d y}{\int_{0}^{y^{*}} R_{h} d y}\right) d y .
$$

Relation (3) is interpreted as the boundary condition of Robin-type obtained by the transfer of the boundary condition from the wall $(y=0)$ to some point $y^{*}$. This boundary condition can be either exact (if the exact function $\mu$ is used in (4)) or approximate (if $\mu$ is approximated by one way or another). One should emphasize that the Robintype boundary condition is simultaneously set for both a function and its derivative. It is easy to see that the mesh distribution nearby the wall can be independently chosen on the location of the value $y^{*}$. Implementation of Robintype conditions to both finite-difference and finite-volume schemes are considered further.

Having assumed that the coefficient varies piece-wise linearly

$$
\mu= \begin{cases}\mu_{w}, & \text { if } 0 \leqslant y \leqslant y_{v}, \\ \mu_{w}+\left(\mu^{*}-\mu_{w}\right) \frac{y-y_{v}}{y^{*}-y_{v}}, & \text { if } y_{v} \leqslant y \leqslant y^{*},\end{cases}
$$

it is possible to obtain analytical expressions for $f_{1}$ and $f_{2}$ if $R_{h}=$ const and $y_{v} \leqslant y^{*}$ :

$$
f_{1}=\alpha_{\mu} y_{v}\left(1+\theta \ln \alpha_{\mu}\right), \quad f_{2}=\alpha_{\mu} y_{v}\left[(1-\theta) y^{*}+y_{v}\left(\theta^{2} \alpha_{\mu} \ln \alpha_{\mu}-1 / 2+\theta\right)\right]
$$

where $\alpha_{\mu}=\mu^{*} / \mu_{w}, \theta^{-1}=\frac{\mu^{*}-\mu_{w}}{\mu_{w}} \frac{y_{v}}{y^{*}-y_{v}}$. Here, the value $y_{v}$ is the thickness of the viscous sublayer near the wall; the parameter $\theta$ represents cotangent of the inclination angle of the dependence $\mu / \mu_{w}$ on $y / y_{v}$.

Assume now that the governing equations correspond to the Reynolds averaged Navier-Stokes equations (RANS) closed by the HR $k-\epsilon$ model. Then, the method of boundary condition transfer technique can be used to derive the wall functions for the tangential and normal velocity components, the temperature and the turbulent kinetic energy.

Having neglected diffusion parallel to the wall, the momentum, enthalpy and kinetic energy transport equations can be written in the Cartesian coordinate system $(x, y)$ :

$$
\begin{aligned}
& \frac{\partial}{\partial y}\left[\left(\mu_{l}+\mu_{t}\right) \frac{\partial U}{\partial y}\right]=\rho U \frac{\partial U}{\partial x}+\rho V \frac{\partial U}{\partial y}+\frac{\partial P}{\partial x}, \\
& \frac{\partial}{\partial y}\left[\left(\mu_{l}+\mu_{t}\right) \frac{\partial V}{\partial y}\right]=\rho U \frac{\partial V}{\partial x}+\rho V \frac{\partial V}{\partial y}+\frac{\partial P}{\partial y}, \\
& \frac{\partial}{\partial y}\left[\left(\frac{\mu_{l}}{P r}+\frac{\mu_{t}}{P r_{t}}\right) \frac{\partial T}{\partial y}\right]=\rho U \frac{\partial T}{\partial x}+\rho V \frac{\partial T}{\partial y}, \\
& \frac{\partial}{\partial y}\left[\left(\mu_{l}+\frac{\mu_{t}}{P r_{k}}\right) \frac{\partial k}{\partial y}\right]=\rho U \frac{\partial k}{\partial x}+\rho V \frac{\partial k}{\partial y}-P_{k}+\rho \epsilon .
\end{aligned}
$$

Here $\mu_{l}$ and $\mu_{t}$ are the laminar and turbulent viscosities, respectively; $\operatorname{Pr}, \operatorname{Pr}_{t}$ and $\operatorname{Pr}_{k}$ are Prandtl numbers; $U$ and $V$ are the velocity components in the $(x, y)$ coordinate system; $\rho$ is the density; $P$ is the pressure; $T$ is the temperature; $k$ is the turbulent kinetic energy; $P_{k}$ is its production; $\epsilon$ is the dissipation of $k$.

Thus, the governing equations for the considered variables have form (1). Evidently, the efficient coefficient $\mu$ in (1) must take into account the appropriate Prandtl number [29]. Upon substitution $U, V, T$ or $k$ instead of $u$ in (3), we obtain RWF for these functions. The right-hand side $R_{h}$ can be evaluated at $y=y^{*}$ as follows [29]:

$$
\begin{aligned}
& R_{h}=R_{h u} \equiv \rho\left(U \frac{\partial U}{\partial x}\left(y^{*}\right)+V \frac{\partial U}{\partial y}\left(y^{*}\right)\right)+\frac{\partial P}{\partial x}\left(y^{*}\right), \\
& R_{h}=R_{h v} \equiv \rho\left(U \frac{\partial V}{\partial x}\left(y^{*}\right)+V \frac{\partial V}{\partial y}\left(y^{*}\right)\right)+\frac{\partial P}{\partial y}\left(y^{*}\right), \\
& R_{h}=R_{h t} \equiv \rho\left(U \frac{\partial T}{\partial x}\left(y^{*}\right)+V \frac{\partial T}{\partial y}\left(y^{*}\right)\right), \\
& R_{h}=R_{h k} \equiv \rho\left(U \frac{d k}{d x}\left(y^{*}\right)+V \frac{d k}{d y}\left(y^{*}\right)\right)+\rho \epsilon-\mu_{t}\left(\frac{d U}{d y}\right)^{2} .
\end{aligned}
$$


Thus, all the terms of the parabolized (reduced) Navier-Stokes equations (PNS) [22] are taken into account in RWF. It is worth noting that in RWF for the normal velocity it is not assigned to be zero. Thus, these wall functions do not have direct restrictions to their exploration in modeling separated flows.

In contrast to the other functions, the right-hand side $R_{h}=R_{h k}$ for the turbulent kinetic energy is variable. It includes the dissipation $\epsilon$ and derivative $d U / d y$. The former term is taken from (17) while the latter term can be evaluated in the interval $\left[0, y^{*}\right][31]$ by:

$$
\left(\mu_{l}+\mu_{t}\right) d U / d y=\left[\left(\mu_{l}+\mu_{t}^{*}\right) U\left(y^{*}\right)+f 2 R_{h u}\right] / f 1+\left(y-y^{*}\right) R_{h u},
$$

where the turbulent viscosity $\mu_{t}$ is approximated by a piece-wise function as in [7,8]:

$$
\mu_{t}= \begin{cases}0, & \text { if } 0 \leqslant y \leqslant y_{v}, \\ \mu_{t}^{*} \frac{y-y_{v}}{y^{*}-y_{v}}, & \text { if } y_{v} \leqslant y \leqslant y^{*} .\end{cases}
$$

In distinguish to all the other variables, $\epsilon$ is set by a Dirichlet boundary condition [8]:

$$
\epsilon(y)= \begin{cases}\frac{\left(k^{*}\right)^{3 / 2}}{C_{l} y_{d}}, & \text { if } y<y_{d}, \\ \frac{\left(k^{*}\right)^{3 / 2}}{C_{l} y}, & \text { else, }\end{cases}
$$

where $y_{d}=2 C_{l} \mu_{l} /\left(\rho \sqrt{k^{*}}\right)$.

RWF include the sublayer thickness $y_{v}$ which is evaluated as follows [29]:

$$
y_{v}=\operatorname{Re}_{v} \mu_{l} /\left(\rho \sqrt{k^{*}}\right),
$$

where $R e_{v}=\rho \sqrt{k^{*}} y_{v} / \mu_{l}$.

The value $R e_{v}$ is varied between 10.8 and 20 [8,3]. The boundaries of this interval correspond to the upper limit of the viscous sublayer and the point at which the linear and logarithmic parts of the velocity profile intersect for the channel flow [20]. In all computational results given below, $R e_{v}=12$ only for sake of determination.

The coefficients $f_{1}$ and $f_{2}$ in wall functions (3)-(6) depend only on $y^{*}$ and $k^{*}$. The latter value is determined from the solution of the HR model at the boundary point $y^{*}$. Hence, the intermediate boundary conditions (3) at $y=y^{*}$ complete the boundary value problem in the interval $\left[y^{*}, y_{e}\right]$. Thus, the developed wall functions can be applied to all dependent variables but $\epsilon$ in a uniform manner. It is worth noting that the coefficients $f_{1}$ and $f_{2}$ can be determined analytically even in the case of the turbulent kinetic energy $k$. Since the laminar sublayer is not resolved proper in the approach based on the piece-wise approximation (5), one cannot expect the entire consistence of RWF (as any other wall functions) with the LR model. Meanwhile, the coefficients $f_{1}$ and $f_{2}$ can also be determined by integrating LR equations in the interval $\left[0, y^{*}\right]$ [29]. It results in a decomposition method. Although the $k-\epsilon$ model is only considered here, it is clear that the same approach to the construction of RWF can be applied to any other turbulent model.

It is to be noted that RWF utilize the assumption on the near-wall direction is predominant in the flow. In some areas, for example, in the vicinity of the axis of symmetry considered further, this assumption can be violated. Meanwhile, the same wall functions might formally be used there. A similar case appears for the Parabolized Navier-Stokes equations [22], and RWF are based on this model.

\section{Robin-type wall functions and the theory of potentials}

There is a deep relation between the Robin-type wall functions and the theory of Calderon-Ryaben'kii potentials $[23,24]$ briefly described below.

Let us consider some domain $D^{0} \subset \mathbb{R}^{n}$ and subdomain $D \subset D^{0}$ having a boundary $\Gamma$, and set the following boundary value problem:

$$
L_{D^{0}} U=F, \quad U \in \Xi_{D^{0}} .
$$

Assume that the space $\Xi_{D^{0}}$ such that the solution of problem (19) exists and unique.

Then, we introduce a potential as follows [23]:

$$
P_{D} V_{D}(\mathbf{x}) \stackrel{\text { def }}{=} V_{D}-\int_{D} G(\mathbf{x}, \mathbf{y}) L V(\mathbf{y}) d \mathbf{y} .
$$


Here and further, $V_{D}$ is the value of some function $V \in \Xi_{D^{0}}$ on $\bar{D} ; G$ is Green's function of the boundary value problem (19). It is to be noted that its value does not depend on the complementation of $V_{D}$ to any function $V^{\prime} \in \Xi_{D^{0}}$.

It is possible to prove [23] that the potential satisfies the homogeneous equation in the domain $D: L_{D^{0}} P_{D} V_{D \mid D}=$ $0_{D}$.

In [23], it is introduced the notion of a clear trace $\operatorname{Tr}_{\Gamma} U_{D}$ in such a way that:

$$
\operatorname{Tr}_{\Gamma} V_{D}=\operatorname{Tr}_{\Gamma} W_{D} \quad \Rightarrow \quad P_{D} V_{D}=P_{D} W_{D}
$$

if $V \in \Xi_{D^{0}}, W \in \Xi_{D^{0}}$.

Then, it is possible to introduce the potential with a density $\xi_{\Gamma}$ :

$$
P_{D \Gamma} \xi_{\Gamma} \stackrel{\text { def }}{=} P_{D} V_{D}
$$

where $\xi_{\Gamma}=\operatorname{Tr}_{\Gamma} V_{D}$.

In [23], it is proved that the following equality

$$
\xi_{\Gamma}=\operatorname{Tr}_{\Gamma} P_{D \Gamma} \xi_{\Gamma}+\operatorname{Tr}_{\Gamma} \int_{D} G(\mathbf{x}, \mathbf{y}) F_{\mid D}(\mathbf{y}) d \mathbf{y}
$$

provides a necessary and sufficient condition for $\xi_{\Gamma}$ to be a clear trace of a function $V_{D}$ :

$$
L_{D^{0}} V_{\mid D}=F_{\mid D}
$$

It is to be noted that equality (23) applied to an external boundary value problem, for example in solving the NavierStoked equations, leads to artificial nonlocal boundary conditions on an artificial boundary [26]. These boundary conditions are formulated in the form of a pseudo-differential boundary equation

$$
{\frac{\partial U}{\partial \mathbf{n}_{\mid \Gamma}}}=A_{\Gamma} U_{\Gamma}
$$

where $A_{\Gamma}$ is a nonlocal operator of Poincaré-Steklov type. As noted in [26], the exact transfer of boundary conditions from one boundary to another (artificial) boundary must lead to nonlocal boundary conditions of the kind of (25). Another particular class of the Poincaré-Steklov operators are provided by the Dirichlet-to-Neumann (DtN) maps $[16,12,13]$.

It is also worth noting that although Green's function is used in the definition of the potential (20), (22), in practical applications explicit Green's function is not required. Instead, its use is efficiently replaced by the Green operator $\widehat{G}_{D^{0}}$ to be inverse to the operator $L_{D^{0}}$.

Let us now suppose that the interval $\left[0, y^{*}\right],\left(y^{*}<y_{e}\right)$ corresponds to the domain $D \subset D^{0}$ where $D^{0}:=\left[0, y_{e}\right]$.

Assume that in the interval $\left[0, y^{*}\right]$ the operator $L_{D^{0}}$ coincides with the differential operator $L$ of Eq. (1). Suppose that we have the following boundary condition on the left-hand side:

$$
l u(0)=\alpha_{0} .
$$

Here $l$ is a local operator.

We introduce the following function of $v$ at the boundary $\Gamma$ :

$$
\operatorname{Tr}_{\Gamma} v_{D}=\left(\operatorname{lv}(0), v\left(y^{*}\right), \frac{d v}{d y}\left(y^{*}\right)\right)^{T} .
$$

Vector (27) corresponds to a clear trace associated with the potential $P_{D} v_{D}$. To prove this statement, it is enough to demonstrate that if $\operatorname{Tr}_{\Gamma} w_{D}=0$ and $w \in \Xi_{D^{0}}$ then $P_{D} w_{D}=0_{D}$.

Indeed, if $\operatorname{Tr}_{\Gamma} w_{D}=0_{\Gamma}$, then the function $w_{D}$ can smoothly be extended to the entire domain $\overline{D^{0}}$ by the nilfunction:

$$
w(x)= \begin{cases}w_{D} & \text { if } x \in D, \\ 0 & \text { if } x \in \overline{D^{0}} \backslash D .\end{cases}
$$

It is easy to see that $w \in \Xi_{D^{0}}$ and $\int_{D} G(x, y) L w d y=\int_{D^{0}} G(x, y) L w d y=w$. Thus, from the definition, $P_{D} w_{D}=$ $0_{D}$ and vector (27) represents the clear trace. 
To demonstrate the application of the potential theory, let us sequentially consider the cases of homogeneous and nonhomogeneous equations (1).

First, introduce into consideration the following boundary value problems in the interval $\left[0, y^{*}\right]$ :

$$
\begin{aligned}
& L u_{1}=0, \\
& l u_{1}(0)=1, \\
& u_{1}\left(y^{*}\right)=0,
\end{aligned}
$$

and

$$
\begin{aligned}
& L u_{2}=0, \\
& l u_{2}(0)=0, \\
& u_{2}\left(y^{*}\right)=1 .
\end{aligned}
$$

1. Assume now that $R_{h} \equiv 0$. Then, from (23)

$$
\xi_{\Gamma}=\operatorname{Tr}_{\Gamma} P_{D \Gamma} \xi_{\Gamma}
$$

We represent the function $u$ as follows:

$$
u=\alpha_{0} u_{1}+u\left(y^{*}\right) u_{2} .
$$

If $\xi_{\Gamma}=\left(l u(0), u\left(y^{*}\right), \frac{d u}{d y}\left(y^{*}\right)\right)^{T}$, then in equality (30) the first two equations are automatically valid. The third equation gives us RWF:

$$
u^{\prime}\left(y^{*}\right)=\alpha_{0} u_{1}^{\prime}\left(y^{*}\right)+u\left(y^{*}\right) u_{2}^{\prime}\left(y^{*}\right) .
$$

It is worth noting here that equality (32) is equivalent to the application of Poincaré-Steklov operator to $u\left(y^{*}\right)$ at the interface boundary $y^{*}$.

2. If $R_{h} \neq 0$, then

$$
\xi_{\Gamma}=\operatorname{Tr}_{\Gamma} P_{D \Gamma} \xi_{\Gamma}+\operatorname{Tr}_{\Gamma}\left(G R_{h}\right) .
$$

The Green's function of the problem is given by:

$$
G(x, y)= \begin{cases}\frac{u_{1}(x) u_{2}(y)}{\mu w\left(u_{2}, u_{1}\right)} & \text { if } 0 \leqslant x \leqslant y, \\ \frac{u_{1}(y) u_{2}(x)}{\mu w\left(u_{2}, u_{1}\right)} & \text { if } y \leqslant x \leqslant y^{*},\end{cases}
$$

where $w\left(u_{2}, u_{1}\right) \stackrel{\text { def }}{=} u_{2} u_{1}^{\prime}-u_{2}^{\prime} u_{1}$ is the Wronskian of $u_{2}$ and $u_{1}$. Then, the solution of the following boundary value problem

$$
\begin{aligned}
& L u_{3}=R_{h}, \\
& l u_{3}(0)=0, \\
& u_{3}\left(y^{*}\right)=0
\end{aligned}
$$

is given by convolution $u_{3}=G * R_{h}$ or:

$$
u_{3}(x)=\frac{1}{\mu(0) w(0)}\left[u_{1}(x) \int_{0}^{x} u_{2}(x) R_{h}(y) d y+u_{2}(x) \int_{x}^{y^{*}} u_{1}(y) R_{h}(y) d y\right] .
$$

Here, we used the Liuville identity: $\mu w=$ const.

Then, the function $u$ is now represented by

$$
u=\alpha_{0} u_{1}+u\left(y^{*}\right) u_{2}+u_{3} .
$$

Since $l u_{3}(0)=0$ and $u_{3}\left(y^{*}\right)=0$, then the first two equations in (33) are valid. 
The last equation gives us the following RWF:

$$
\begin{aligned}
u^{\prime}\left(y^{*}\right) & =\alpha_{0} u_{1}^{\prime}\left(y^{*}\right)+u\left(y^{*}\right) u_{2}^{\prime}\left(y^{*}\right)+u_{3}^{\prime}\left(y^{*}\right) \\
& =\left(\alpha_{0}+\frac{1}{\mu(0) w(0)} \int_{0}^{y^{*}} u_{2}(y) d y R_{h}(y) d y\right) u_{1}^{\prime}\left(y^{*}\right)+u\left(y^{*}\right) u_{2}^{\prime}\left(y^{*}\right) .
\end{aligned}
$$

If the boundary condition on the left-hand side corresponds to (2), then

$$
\begin{aligned}
& u_{1}(y)=1-C_{0} \int_{0}^{y} \frac{d x}{\mu}, \\
& u_{2}(y)=C_{0} \int_{0}^{y} \frac{d x}{\mu}, \\
& \mu(0) w(0)=C_{0}, \\
& C_{0}^{-1}=\int_{0}^{y^{*}} \frac{d x}{\mu}
\end{aligned}
$$

and

$$
u\left(y^{*}\right)=u_{0}+f_{1} u^{\prime}\left(y^{*}\right)-\frac{1}{\mu_{e}} \int_{0}^{y^{*}}\left(R_{h}(x) \int_{0}^{x} \frac{\mu}{\mu_{e}} d y\right) d x .
$$

This equality fully coincides with (3), (4). The identity of the last term in (40) can be shown via integrating by parts.

According to [23] RWF represent so-called "internal" boundary conditions which are completely independent from the external problem. Since the solution $u$ of boundary value problem (1), (2) is smooth across the intermediate boundary $y^{*}$, the same condition (40), on the function and its normal derivative, is to be set in the "main" external flow.

It is to be noted that all known wall functions including RWF are locally one-dimensional. The representation of RWF in the form of boundary equation (23) opens a way to construct nonlocal wall functions. In multidimensional case (23) leads to a nonlocal relation between the function and its normal derivative represented by a nonlocal pseudodifferential equation. It can be written in the form of (25) or its nonhomogeneous analog. In this case, a clear trace operator is given by the following vector

$$
\operatorname{Tr}_{\Gamma} U_{D}=\left(l U_{\mid \Gamma_{0}}, U_{\mid \Gamma_{c}}, \frac{\partial U}{\partial \mathbf{n} \mid \Gamma_{c}}\right)^{T},
$$

where $\Gamma_{0}:=\partial D^{0}, \Gamma_{c}:=\Gamma_{0} \backslash \Gamma$. This statement can be proved completely similar to the $1 \mathrm{D}$ case considered above. The solution of the nonlocal equation can effectively be realized via the difference potential method by Ryaben'kii [23]. It is to be noted that an important advantage of the Calderon-Ryaben'kii potentials is that they allow one to obtain the boundary equation (23) without the use of Green's function. Thus, they are applicable for RANS models and complex geometries.

\section{Comparative analysis against analytical wall functions}

The analytical wall functions (AWF) and RWF have the same nature since they are derived on the same basic assumptions. In some realizations they should provide identical results.

Meanwhile, there are a number of substantial distinctions. They are mostly related to the fact that AWF are integrated to the finite-volume scheme used while RWF are represented as mesh independent differential Robin boundary conditions. They are not dependable on the approximation used in the external domain and can be incorporated in finite-difference and finite element approximations, as well. 
The incorporation of AWF is equivalent to the replacement of the numerical integration in the near-wall cell by a higher order integration. In particular, for the standard second-order approximations, a piece-wise linear approximation across a computational cell is used for the coefficient $\mu$ instead of the linear approximation. Via the consideration of a differential approximation it is possible to demonstrate that piece-wise approximation (5) leads to a singularity because of the lack of smoothness of the coefficient $\mu$. This might cause a nonstable numerical solution in the application of AWF [8]. This problem was overcome in [8] by the use of a hyperbolic approximation for $\mu$ resulting in a much weak singularity. Meanwhile, in the case of RWF such a problem does not appear because they are based only on a local integration. A numerical differentiation is only used in the external domain where the solution is locally smooth.

Because AWF are incorporated into numerical integration, the value $y^{*}$ is inevitably related to the near-wall mesh distribution. The value of $y^{*}$ cannot substantially differ from the next mesh size in the normal direction, otherwise it affects the approximation and robustness of the algorithm. Meanwhile, RWF are mesh independent, and they are independently formulated from the external problem.

As it follows from the general theorem [23] formulated for the internal boundary conditions, RWF can be used for different external problems, for example in LES. Their formulation does not depend on either the scheme or equations used in the external domain. Hence, they can be realized in a separate routine which can be implemented to different codes. In this case, the two values characterizing a Robin boundary condition are to be transferred from the routine to the main code at each node along a wall.

In addition, RWF are formulated in a unified form for all dependent variables but $\epsilon$. In the case of linear equations, their realization does not require any iterations. Therefore, their realization for nonlinear problems does not bring any additional iterations into the entire iteration process.

As shown in the previous section, RWF can be derived via the theory of Calderon-Ryaben'kii's potentials. This provides an opportunity to generalize them to the nonlocal formulation having the form of boundary equation (25) or its nonhomogeneous analog.

\section{Numerical implementation of RWF}

RWF can be implemented to both finite-difference and finite-volume RANS approximations. In this section, some aspects of their robust implementation are considered.

A general remark related with robust implementation is the following. Boundary conditions of Robin-type are set on both a function and its derivative. Upon approximating the derivative, both terms should be considered at the same iteration (or time step). Taking into account one of the terms from a previous iteration leads to additional iterations, at least. It is easy to see this property in the case of a linear equation. Only simultaneous consideration of both terms provides an iterationless solution.

It is to be noted that the boundary condition is represented by (3) for any $y^{*}$ in the vicinity of the wall even if $y^{*}$ vanishes [29]. This boundary condition fully replaces the original boundary condition $u(0)=u_{0}$.

In numerical simulation of turbulence, the finite-difference numerical schemes preserving positiveness of a solution [18] are very efficient because unknown variables such as the turbulent kinetic energy $k$ or its dissipation $\epsilon$ must be positive. The following numerical procedure can be used for developing the positive definite schemes in solving boundary-value problems with Robin-type boundary conditions [29].

Boundary condition (3) can be rewritten in the following general form:

$$
u\left(y^{*}\right)=\alpha d u / d y\left(y^{*}\right)+\beta,
$$

where both the function $u$ and its derivative $d u / d y$ are positive. It is valid in the case of real physical problems for the turbulent kinetic energy in the wall vicinity. The coefficient $\alpha$ is positive because $f_{1}$ is always positive but the coefficient $\beta$ can be negative (mostly, where $\epsilon>P_{k}$ ). In computations it can lead to a negative value of $u$. To avoid such a case, it is suggested to rewrite (42) in the following form if $\beta<0$ :

$$
u\left(y^{*}\right)=\alpha d u / d y\left(y^{*}\right)+\beta \frac{u\left(y^{*}\right)}{u^{-}\left(y^{*}\right)},
$$

or

$$
u\left(y^{*}\right)=\tilde{\alpha} d u / d y\left(y^{*}\right),
$$

where $\tilde{\alpha}=\frac{\alpha}{1-\beta / u^{-}\left(y^{*}\right)}$ and $u^{-}\left(y^{*}\right)$ is the value of $u\left(y^{*}\right)$ taken from the previous either time step or iteration. 
Although Robin-type boundary condition (3) can be set at the wall, its implementation to existing codes based on finite-volume schemes is more easy in the following interpretation. Assume that unknown variables are defined at the centers of cells. For sake of simplicity let us consider 1D approximation in the normal to the wall direction. It is enough to consider approximation at the nearest to the wall cell since the rest approximation is remained without any modification. Let us denote values at the center of the cell by index $1 / 2$ and values at the edge, opposite to the wall, by index 1 . Then, considering $y^{*}=y_{1 / 2}$ we have a relation in the following form:

$$
u_{1 / 2}=u_{w}+f_{1} \frac{d u}{d y}+1 / 2=\tilde{f}_{2},
$$

where $\tilde{f}_{2}=-\frac{\int_{0}^{y_{1 / 2}} R_{h} d y}{y_{1 / 2} \mu\left(y_{1 / 2}\right)} f_{2}$. At the first cell the governing equation (1) is then integrated only from $y_{1 / 2}$ to $y_{1}$ :

$$
\mu \frac{d u}{d y_{\mid 1}}-\mu \frac{d u}{d y_{\mid 1 / 2}}=\int_{y_{1 / 2}}^{y_{1}} R_{h} d y .
$$

Eq. (45) represents the approximation of the governing equation in the near-wall cell. Here, the flux $\mu \frac{d u}{d y}{ }_{\mid 1}$ is approximated by an ordinary approach while the flux at $y_{1 / 2}$ is obtained from (44). A typical approximation can be written as follows:

$$
\mu_{1} \frac{u_{3 / 2}-u_{1 / 2}}{y_{1}}-\mu_{1 / 2} \frac{u_{1 / 2}-u_{w}-\tilde{f}_{2}}{f_{1}}=\int_{y_{1 / 2}}^{y^{1}} R_{h} d y .
$$

This kind of approximation is used for all the variables, but $\epsilon$, including $k$. In the last case, the right-hand side is rapidly changed and the integral in the right-hand side must be evaluated accurately enough. It can be easily done either numerically or analytically using the analytical expression for the velocity gradient (15).

At last, a brief comment can be done with regard to a staggered mesh. In this approach the velocity is defined at the vertexes of a cell. In our consideration this means $u_{1}$ is known instead of $u_{1 / 2}$. In this case, the simplest way to remain the uniform approach is based on the Taylor expansion:

$$
u_{1}=u_{1 / 2}+y_{1 / 2} \frac{d u}{d y}_{\mid 1 / 2}+\frac{y_{1 / 2}^{2}}{2}{\frac{d^{2} u}{d y^{2}}}_{\mid 1 / 2} .
$$

This yields a required relation between $u_{1 / 2}$ and $u_{1}$ since the first derivative takes place in (44) and the second derivative can be evaluated via the right-hand side $R_{h}$. Thus, the flux at the nearest to the wall cell is as follows:

$$
\mu_{1 / 2}{\frac{d u}{d y_{\mid 1 / 2}}}=\mu_{1 / 2} \frac{u_{1}-u_{w}}{f_{1}+h / 2}-R_{h} \frac{h^{2} / 8-\tilde{f}_{2}}{f_{1}+h / 2} .
$$

The same technique with slight modifications can be used for implementation to unstructured codes.

\section{Impinging jet test case}

The heat transfer problem on a turbulent circular jet impinging onto a flat is well studied experimentally [6,2,31]. Therefore, it became a widespread test case for different turbulent models including the LR $[9,25,14,10,21]$ and HR $k-\epsilon$ models $[11,14,1]$.

The problem is formulated as follows. A fully turbulent air jet, generated in a pipelike nozzle, impinges on a flat surface at the right angle. The Reynolds number, based on the nozzle diameter $D$ and the bulk velocity, equals to $R e=23000$ and $R e=70000$. The distance $L$ between the nozzle and the surface is varied between $2 D$ and $14 D$. Air is treated as an ideal gas and considered under normal conditions at temperature $293 \mathrm{~K}$. The heated surface has constant temperature $T_{w}=314.9 \mathrm{~K}$.

The computational domain spans $13 D$ in the radial direction. The grid includes $150 \times 100$ (axial $\times$ radial) nodes and $150 \times 200$ nodes. For the validation purposes, preliminary comparisons of the results obtained on different meshes were done to check grid sensitivity. The boundary conditions at the edge of the nozzle are specified using the profiles 
for a fully developed turbulent pipe flow. The computations of the local Nusselt number are done for the different values of $y^{*}$ or $\operatorname{Re}_{y^{*}} \equiv \rho \sqrt{k^{*}} y^{*} / \mu_{l}$ calculated at the stagnation point.

It is well known that linear eddy-viscosity models (EVM) drastically overpredict the turbulent kinetic energy in the stagnation point region by an order of magnitude [1]. It inevitably leads to the considerable overestimation of the heat flux. As a result, the linear LR $k-\epsilon$ models give unacceptable overprediction by a factor of two, even more $[21,25]$. Furthermore, the linear $k-\epsilon$ model, as well as other EVM, is not entirely justified around the stagnation point because of the anisotropy of the flow. To improve prediction, along with the nonlinear EVM, some modifications of the EVM can be used.

It is important to note that the primary goal of the current consideration contains numerical aspects of RWF implementation rather than the justification of the turbulence model used. It includes robustness of the computational algorithm, weak dependence of the solution on the near-wall mesh distribution and applicability of RWF to different operating regimes without any free parameters.

RWF are applied in [29] to simulation of the impinging jet at $R e=23000$ with $L / D=2$ and $L / D=6$. Apart from the heat flux, the prediction of the wall friction is considered in [29]. As noted above, the overprediction of the heat flux was obtained in the computational solution. The same effect but more expressive is observed at $R e=70000$. In Fig. 1, the computational results are compared against the experimental data for $L / D=2$. Here and below, the local Nusselt number is scaled by $\operatorname{Re}{ }^{0.7} \operatorname{Pr}^{0.4}$ where $\operatorname{Pr}=0.9$. The solution [9] based on the LR $k-\epsilon$ model predicts a substantially higher heat flux than the HR model. This effect was obtained in many other publications including cited above. The solution based on the wall functions corresponds to $R e_{y^{*}}=109$.

The HR model with RWF provides much better prediction than the LR model in the vicinity of the axis of the symmetry. The explanation of this, at first glance, counterintuitive result is as follows. The overprediction of the isotropic LR model is related to the high production of the turbulent kinetic energy $P_{k}$ in this area characterized by high anisotropy of the flow. RWF are based on the parabolized Navier-Stokes equations and take into account only the wall shear stress component of the stress tensor in the production term $P_{k}$ included in (14). Thus, the inconsistent terms of the LR model appear to be not included in the RWF model. A similar situation arises in the problem of heat transfer in the vicinity of a stagnation point for hypersonic gas flow over a blunt body. At very low Reynolds numbers the asymptotic viscous shock layer equations provide results well consistent with the free-molecular regime, while the full Navier-Stokes equations completely fail [5,4].

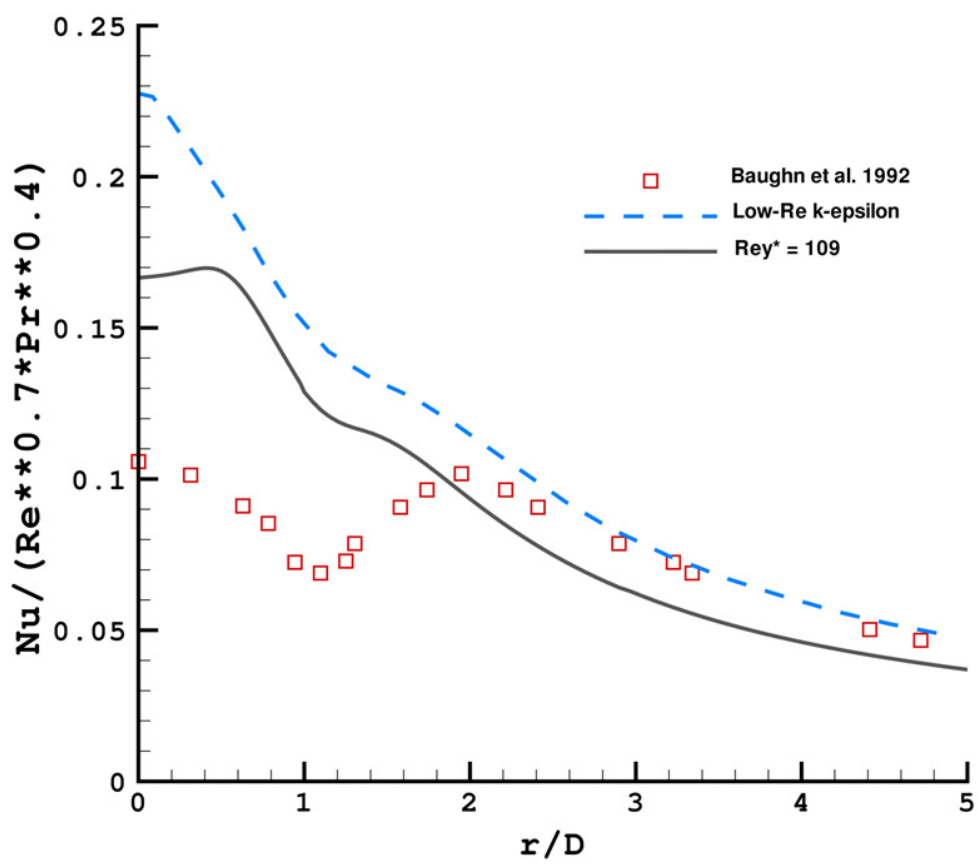

Fig. 1. Local Nusselt number for the impinging jet. Comparison between HR, LR solutions and experiment for $R e=70000$ and $L / D=2$. 


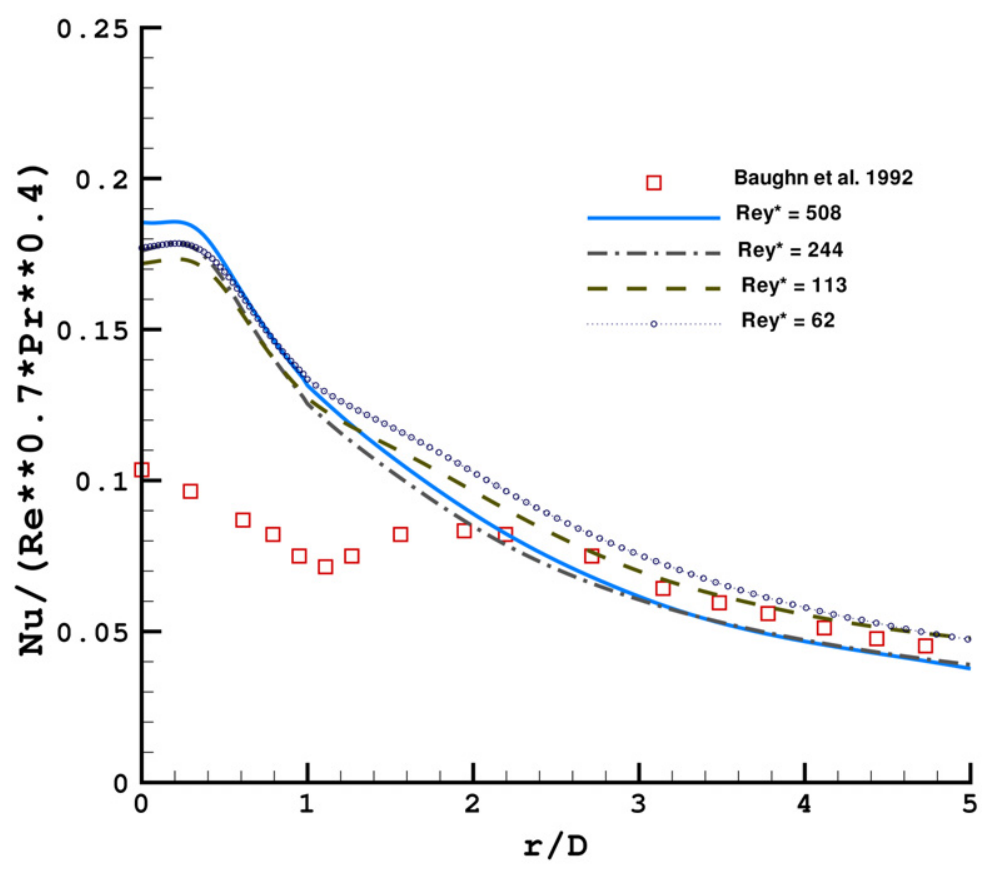

Fig. 2. Local Nusselt number for the impinging jet. Comparison of computational solution for different $y^{*}$ against experiment for $R e=70000$ and $L / D=4$.

In the next example with $L / D=4$, the solutions corresponding to different values of $R e_{y^{*}}$ are shown in Fig. 2. Although the value of $y^{*}$ is varied by an order of magnitude, the curves are quite close each other. It is to be noted that the dependence of the solution on the parameter $y^{*}$ is quite weak.

The mean velocity profiles divided by the bulk velocity are shown in Fig. 3 for $L / D=6$. The experimental data are represented by square symbols while the computational results are shown by the curves. At the region of the low mean velocity nearby the axis of symmetry $(r / D=0.5)$ the prediction of the velocity is quite accurate. At $r / D=3$, where the flow is decelerated, the prediction is not so good. At this location, substantial underprediction of the velocity in the near wall region and overprediction in the outer region were earlier noted for both the LR and HR linear $k-\epsilon$ models $[11,9]$.

In Fig. 4 the distribution of the local Nusselt number is shown for $R e=23000$. It is given a comparison between the computational results and experimental data for $L=10 D$ and $L=14 D$. In contrast to the previous examples considered, in these regimes the wall is located far enough from the nozzle, and the overprediction of the heat flux nearby the axis of symmetry is not observed.

\section{Conclusion}

A general robust approach to implementing RWF into finite-volume algorithms has been suggested. RWF are derived in a unit manner for all leading variables. The computational algorithm can be easily realized in existing finite-volume codes. In addition, RWF can be implemented in a separate routine which can be used for different codes and models. The relation between RWF and the theory of Calderon-Ryaben'kii potentials has been shown. This provides a way to the generalization of RWF on a new type of nonlocal wall functions.

The performance of RWF has been considered for the impinging jet test case. The computational results demonstrate a reasonable correspondence to the experimental data and weak dependence of the solution on the distance from the wall to the point, where the boundary conditions are set, for the wide variation of geometries and operating regimes.

Further development will focus on the implementation of RWF in LES and the extension of the RWF strategy to complex geometries (nonlocal wall functions). 


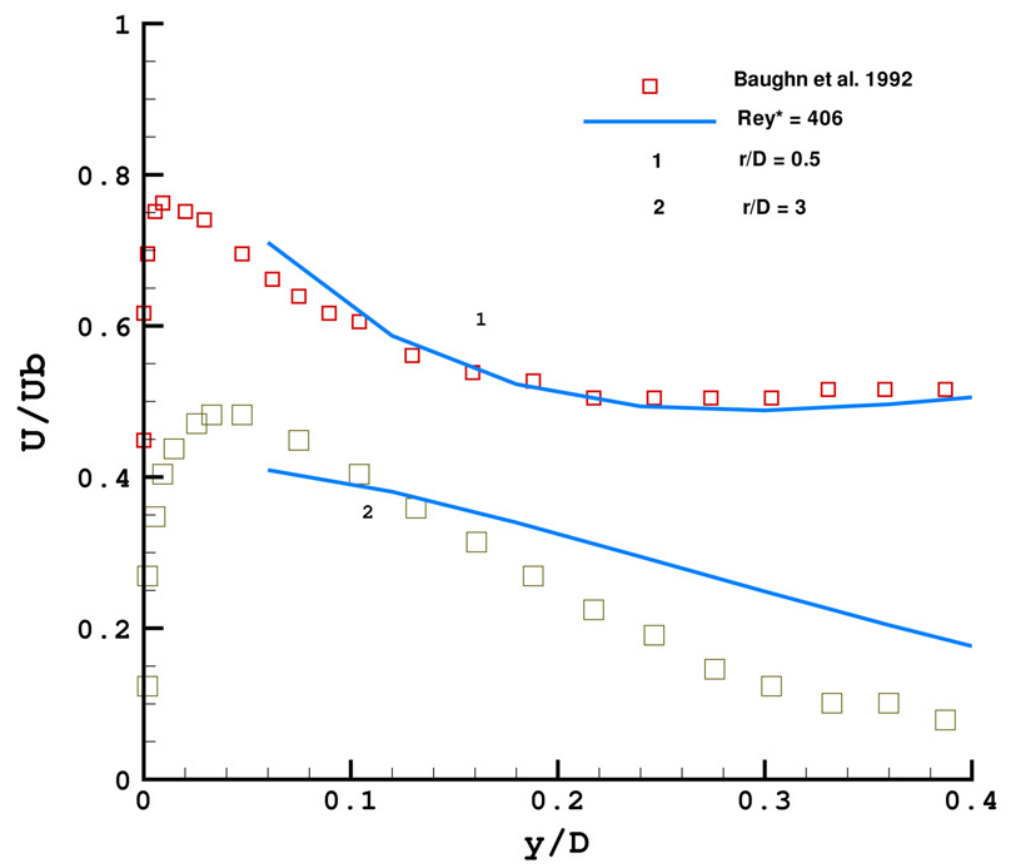

Fig. 3. Mean velocity in the impinging jet at $r / D=0.5 ; 3$. Comparison of computational solution against experimental data for $R e=70000$ and $L / D=6$.

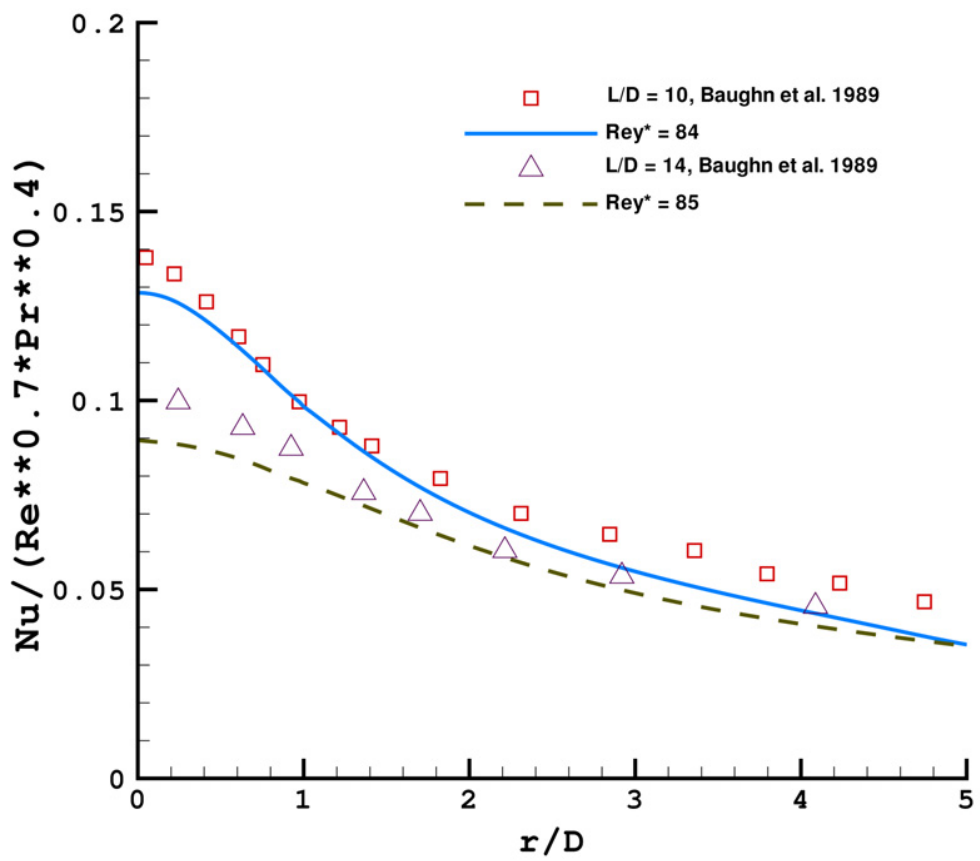

Fig. 4. Local Nusselt number for the impinging jet. Comparison computational solutions against experimental data for $R e=23000$ and $L / D=10 ; 14$.

\section{Acknowledgement}

The author is grateful to the unknown referees for valuable remarks. 


\section{References}

[1] A. Ashforth-Frost, K. Jambunathan, Numerical prediction of semi-confined jet impingement and comparison with experimental data, Int. J. Numer. Methods Fluids 23 (1996) 295-306.

[2] J. Baughn, S. Shimizu, Heat transfer measurements from a surface with uniform heat flux and an impinging jet, Heat Transfer 111 (1989) 1096-1098.

[3] J. Bredberg, S.-H. Peng, L. Davidson, On the wall boundary conditions for computing turbulent heat transfer with $K-\omega$ models, in: J.H. Kim (Ed.), Proceedings of the ASME Heat Transfer Division, vol. 5, Orlando, USA, Nov. 5-10, 2000, 243-250.

[4] I.G. Brykina, B.V. Rogov, G.A. Tirskiy, Continuum models of rarefied gas flows in problems of hypersonic aerothermodynamics, J. Appl. Math. Mech. 70 (6) (2006) 888-911.

[5] H.K. Cheng, The blunt body problem in hypersonic flow at low Reynolds number, in: IAS Paper, No. 63-92, 1963.

[6] D. Cooper, D.C. Jackson, B.E. Launder, G.X. Liao, Impinging jet studies for turbulence model assessment-I. Flow-field experiments, Int. J. Heat Mass Transfer 36 (10) (1993) 2675-2684.

[7] T.J. Craft, S.E. Gant, A.V. Gerasimov, H. Iacovides, B.E. Launder, Wall-function strategies for use in turbulent flow CFD, in: Proceedings of 12th International Heat Transfer Conference, Grenoble, France, 2002, pp. 1-12.

[8] T.J. Craft, A.V. Gerasimov, H. Iacovides, B.E. Launder, Progress in the generalization of wall-functions treatments, Heat and Fluid Flow 23 (2) (2002) 148-160.

[9] T.J. Craft, L.J.W. Graham, B.E. Launder, Impinging jet studies for turbulence model assessment-II. An examination of four turbulence models, Int. J. Heat Mass Transfer 36 (10) (1993) 2685-2697.

[10] T.J. Craft, H. Iacovides, J.H. Yoon, Progress in the of the non-linear two-equation models in the computation of convective heat transfer in impinging and separated flows, Flow, Turbulence and Combustion 63 (1999) 59-80.

[11] T. Esch, F.R. Menter, Heat transfer predictions based on two-equation turbulence models with advanced wall treatment, in: Proceedings of the 4th International Symposium on Turbulence, Heat \& Mass Transfer, Antalya, Turkey, 2003, pp. 614-621.

[12] D. Givoli, Numerical Methods for Problems in Infinite Domains, Elsevier, Amsterdam, 1992.

[13] D. Givoli, I. Patlashenko, Dirichlet-to-Newmann boundary conditions for time-dependent dispersive waves in three-dimensional guides, J. Comput. Phys. 199 (2004) 339-354.

[14] K. Heyerichs, A. Pollard, Heat transfer in separated and impinging turbulent flows, Int. J. Heat Mass Transfer 39 (12) (1996) $2385-2400$.

[15] G. Kalitzin, G. Medic, G. Iaccarino, P.A. Durbin, Near-wall behavior of RANS turbulence models and implications for wall function, J. Comput. Phys. 1 (2005) 265-291.

[16] J.B. Keller, D. Givoli, Exact non-reflecting boundary conditions, J. Comput. Phys. 82 (1989) 172-192.

[17] T. Kopp, On grid-independence of RANS predictions for aerodynamic flows using model-consistent universal wall-functions, in: Proceedings of ECCOMAS CFD'2006, The Netherlands, 2006.

[18] L.W.L. Lanerolle, Positivity preserving pointwise implicit schemes with application to turbulent compressible flat plate flow, Int. J. Numer. Methods Fluids 35 (2001) 903-938.

[19] B. Mohammadi, G. Puigt, Wall functions in computational fluid mechanics, Computers \& Fluids 35 (10) (2006) $1108-1115$.

[20] R. Moser, J. Kim, N. Mansour, Direct numerical simulation of the turbulent channel flow up to $R e=590$, J. Phys. Fluids 11 (1999) $943-945$.

[21] T.S. Park, H.J. Sung, Development of a near-wall turbulence model and application to jet impingement heat transfer, Int. J. Heat Fluid Flow 22 (2001) 10-18.

[22] S.G. Rubin, J.C. Tannehill, Parabolized/Reduced Navier-Stokes computational techniques, Annu. Rev. Fluid Mech. 24 (1992) $117-144$.

[23] V.S. Ryaben'kii, Method of Difference Potentials and its Applications, Springer-Verlag, Berlin, 2002.

[24] V.S. Ryaben'kii, S.V. Tsynkov, A Theoretical Introduction to Numerical Analysis, Chapman \& Hall/CRC, 2006 , Chapter 14.

[25] B. Sunden, R. Jia, A. Abdon, Computation of combined turbulent convective and impingement heat transfer, Int. J. Heat Fluid Flow 14 (1) (2004) 116-133.

[26] S.V. Tsynkov, Numerical simulation of problems on unbounded domains. A review, Appl. Numer. Math. 27 (1998) $465-532$.

[27] S.V. Utyuzhnikov, Some new approaches to building and implementation of wall-functions for modeling of near-wall turbulent flows, Computers \& Fluids 34 (7) (2005) 771-784.

[28] S.V. Utyuzhnikov, Generalized wall-functions and their application for simulation of turbulent flows, Int. J. Numer. Methods Fluid 47 (10-11) (2005) 1323-1328.

[29] S.V. Utyuzhnikov, The method of boundary condition transfer in application to modeling near-wall turbulent flows, Computers \& Fluids 35 (10) (2006) 1193-1204.

[30] D.C. Wilcox, Wall matching, a rational alternative to wall functions, AIAA Paper 89-611, Reno, NV, 1989.

[31] X. Yan, J.W. Baughn, M. Mesbah, The effects of Reynolds number on the heat transfer distribution from a flat plate to an impinging jet, ASME HTD 226 (1992) 1-7. 